\title{
Temperature dependence of ${ }^{3} \mathrm{P}_{\mathbf{0}} \mathrm{Pr}^{3+}$ fluorescence dynamics in $\mathbf{Y}_{\mathbf{4}} \mathrm{Al}_{\mathbf{2}} \mathbf{O}_{\mathbf{9}}$ crystals
}

\author{
M. Kaczkan · Z. Boruc $\cdot$ B. Fetlinski • \\ S. Turczynski $\cdot$ M. Malinowski
}

Received: 26 November 2012 / Accepted: 25 April 2013/Published online: 30 May 2013

(c) The Author(s) 2013. This article is published with open access at Springerlink.com

\begin{abstract}
Temperature-dependent emission spectra and fluorescence dynamics profiles have been investigated in $\mathrm{Pr}^{3+}: \mathrm{Y}_{4} \mathrm{Al}_{2} \mathrm{O}_{9}$ crystals in order to better understand the processes responsible for quenching of the praseodymium ${ }^{3} \mathrm{P}_{0}$ emissions. The cross-relaxation transfer rates were experimentally determined as a function of temperature. Using the rate equations formalism, the dynamics of the observed emissions were modeled. Basing on comparison between the measured and calculated decays, the energy transfer rates between $\mathrm{Pr}^{3+}$ ions were evaluated. The role of the backward process in explanation of the complicated character of ${ }^{3} \mathrm{P}_{0}$ decays and its temperature dependence, especially its unexpectedly slow decaying component, were established.
\end{abstract}

\section{Introduction}

Trivalent praseodymium $\left(\mathrm{Pr}^{3+}\right)$ ion is continuously considered as a promising activator for solid state lasers, optical amplifiers, scintillation detectors, sensors, quantum memories, solar converters and various phosphors [1-4]. This is due to various strong emissions, resulting from both inter-configurational $\mathrm{d}-\mathrm{f}$ and intra-configurational $\mathrm{f}-\mathrm{f}$ transitions extending from UV to near infrared wavelengths,

M. Kaczkan · Z. Boruc · B. Fetlinski · M. Malinowski ( $\square)$ Institute of Microelectronics and Optoelectronics,

Koszykowa 75, 00-662 Warsaw, Poland

e-mail: m.malinowski@elka.pw.edu.pl

S. Turczynski

Institute of Electronic Materials Technology,

Wolczynska 133, 01-919 Warsaw, Poland which could be generated in $\operatorname{Pr}^{3+}$-activated media. The energy level diagram of $\mathrm{Pr}^{3+}$ ion indicates that praseodymium materials have advantageous ability to be pumped by the commercially available $\mathrm{GaN}$ blue diodes and lasers [5] via strong ${ }^{3} \mathrm{H}_{4} \rightarrow{ }^{3} \mathrm{P}_{1,2}$ absorption.

It was pointed out that various transitions from the excited ${ }^{3} \mathrm{P}_{0}$ and ${ }^{1} \mathrm{D}_{2}$ states are of interest in $\mathrm{Pr}^{3+}$ systems, among them the ${ }^{3} \mathrm{P}_{0} \rightarrow{ }^{3} \mathrm{~F}_{2}$ and the ${ }^{1} \mathrm{D}_{2} \rightarrow{ }^{3} \mathrm{H}_{4}$ transitions, which are associated with intense red emissions in the $600 \mathrm{~nm}$ region (see energy level diagram in Fig. 1). In several applications, strong, spin-allowed transitions originating from the ${ }^{3} \mathrm{P}_{0}$ level are utilized. It is thus interesting to investigate the effects of concentration and temperature on these emissions in $\mathrm{Pr}^{3+}$-doped $\mathrm{Y}_{4} \mathrm{Al}_{2} \mathrm{O}_{9}$ (abbreviated YAM) crystals. Very few reports [6,7] are available on the spectroscopic properties of this system so far. Previous studies of $\operatorname{Pr}^{3+}$ :YAM concentrated on its basic optical and spectroscopic properties such as the position of $\mathrm{Pr}^{3+}$ energy levels in $4 \mathrm{f}^{2}$ configuration and the multisite character of this system [6,7]. Thus, there are some interesting basic processes such as nonradiative relaxations and multiion processes that need to be explored. Since such processes in phosphor materials often occur via thermal activation, understanding them is vital to elucidate the luminescence mechanisms. In addition, as it was recently demonstrated, the temperature dependence of the $\operatorname{Pr}^{3+}$ fluorescence features can be used to measure temperature [8-10] and is interesting from the application point of view in optical thermometry.

Thus, the purpose of this paper is three-fold: to extend our knowledge on luminescence properties of the new praseodymium system, to get insight into processes of excitation energy distribution after pumping the ${ }^{3} \mathrm{P}_{0}$ level of $\mathrm{Pr}^{3+}$ ions and to study and model the ${ }^{3} \mathrm{P}_{0}$ state dynamics in the function of temperature. 


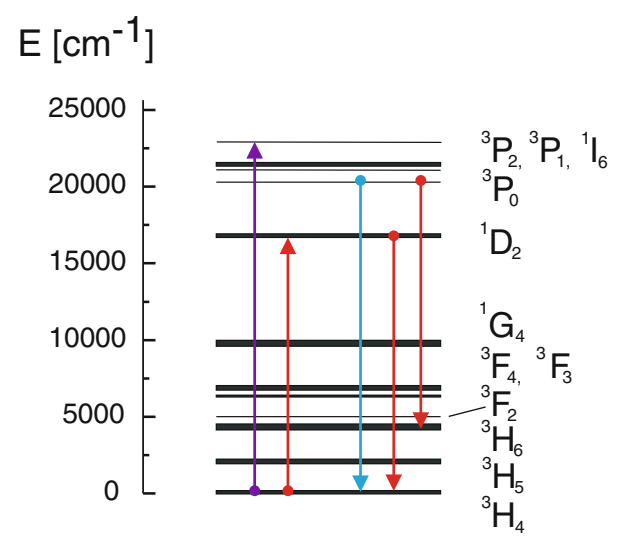

Fig. 1 Generic energy level diagram of $\operatorname{Pr}^{3+}: Y A M$, analyzed transitions are indicated by arrows

\section{Experimental}

Samples used in our study were grown in the Institute of Electronic Materials Technology (ITME) in Warsaw. As YAM undergoes the phase transition at about $1,300{ }^{\circ} \mathrm{C}$ and crystals grown by standard Czochralski method crack during cooling, the micro-pulling down ( $\mu$-PD) method was used to obtain YAM samples. The $\mu$-PD method was invented in Japan, originally for growth of single-crystal fibers [11]. This method was then used in ITME for preparing YAG [12] single crystals and, for the first time to our knowledge, for growing YAM.

Polycrystals in the form of rods 2-3 $\mathrm{mm}$ in diameter and several $\mathrm{cm}$ long were obtained. Four YAM: $\mathrm{Pr}^{3+}$ samples with activator concentrations of $0.1,1,5$ and 10 at.\% were used in our studies.

The yttrium-alumina system has several stable phases including monoclinic $\mathrm{Y}_{4} \mathrm{Al}_{2} \mathrm{O}_{9}$ (YAM), cubic garnet $\mathrm{Y}_{3} \mathrm{Al}_{5} \mathrm{O}_{12}$ (YAG), orthorhombic perovskite $\mathrm{YAlO}_{3}$ (YAP) and a metastable hexagonal perovskite $\mathrm{YAlO}_{3}$ phase (YAH) observed during the synthesis by soft chemistry methods. YAM forms monoclinic crystals with space group $\mathrm{P} 21 / \mathrm{c}$. The $\mathrm{Y}$ atoms, having $\mathrm{C}_{1}$ site symmetry, are coordinated to either six or seven oxygen atoms [13]. The shortest distance between the $\mathrm{Y}^{3+}$ ions is $3.65 \AA$. There are four formula units in the unit cell of $\mathrm{Y}_{4} \mathrm{Al}_{2} \mathrm{O}_{9}$ and four different rare earth sites in the asymmetric unit.

Emission spectra were measured using CVI DK-480 grating monochromator followed by a cooled EMI C1034-02 GaAs photomultiplier and SR-400 photon counting system. The samples were excited by pulsed (10 ns pulse-width, repetition rate $10 \mathrm{~Hz}$ ) Continuum Surelite Nd:YAG laser with third harmonic generator, followed by an optical parametric oscillator, or by $\mathrm{CW}$ Coherent Innova 300 , a $10 \mathrm{~W}$ argon ion laser. Fluorescence dynamics profiles were recorded with Stanford Research SR-430 multi-channel analyzer controlled with a PC computer. The best temporal resolution of the experimental apparatus was $5 \mathrm{~ns}$.

Temperature dependence of the sample fluorescence in the range $300-1,300 \mathrm{~K}$ was measured in a self-made resistive heat cell. The temperature of the samples was monitored by a $\mathrm{Pt} / \mathrm{Rh}$ thermocouple and controlled with accuracy of about $1 \mathrm{~K}$ by Eurotherm PID temperature controller type 3024. Sample cooling was provided by a Displex Model CSW-202 closed cycle He optical cryostat which allowed the temperature to be varied between 10 and $300 \mathrm{~K}$.

\section{Results}

\subsection{Emission}

After excitation of the ${ }^{3} \mathrm{P}_{\mathrm{J}}$ levels by blue-violet laser radiation, rich emission spectrum extending from blue to red wavelength could be observed. These emissions are attributed to transitions originating mostly from the ${ }^{3} \mathrm{P}_{0}$ level. However, also weak fluorescence starting from the ${ }^{1} \mathrm{D}_{2}$ state could be observed in the red-infrared part of the spectrum. Only in the 550-650 $\mathrm{nm}$ range, where emissions from the ${ }^{3} \mathrm{P}_{0}$ and ${ }^{1} \mathrm{D}_{2}$ levels overlap, emission corresponding to the ${ }^{1} \mathrm{D}_{2} \rightarrow{ }^{3} \mathrm{H}_{4}$ transition dominates over ${ }^{3} \mathrm{P}_{0} \rightarrow{ }^{3} \mathrm{~F}_{2}+{ }^{3} \mathrm{H}_{6}$ one. In Fig. 2, part of the emission spectrum in the $600 \mathrm{~nm}$ region after selective excitation is shown. To well distinguish between ${ }^{3} \mathrm{P}_{0}$ and ${ }^{1} \mathrm{D}_{2}$ lines, time resolved selectively excited emission spectra were also recorded at 10 and $300 \mathrm{~K}$ as well as the ${ }^{1} \mathrm{D}_{2} \rightarrow{ }^{3} \mathrm{H}_{4}$ emission spectra following direct excitation into ${ }^{1} \mathrm{D}_{2}$ level.

The observed line positions are consistent with the data reported in the literature for $\operatorname{Pr}^{3+}$ :YAM [6]. In Fig. 3, the temperature-dependent emission measurements of 1 at.\% $\mathrm{Pr}^{3+}$ :YAM in the 560-720 $\mathrm{nm}$ wavelength range after blue ${ }^{3} \mathrm{P}_{2}$ excitation are shown. It is observed that both ${ }^{3} \mathrm{P}_{0}$ and

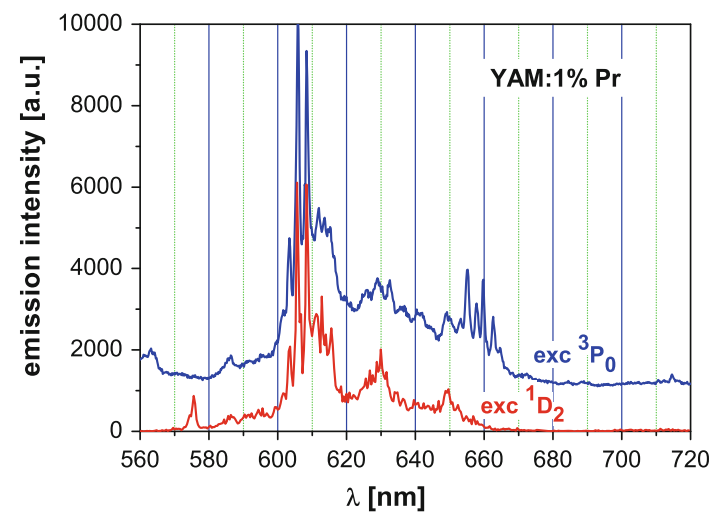

Fig. 2 Emission spectra of $1 \% \operatorname{Pr}^{3+}: Y A M$ in the $600 \mathrm{~nm}$ region, $T=300 \mathrm{~K}$ 


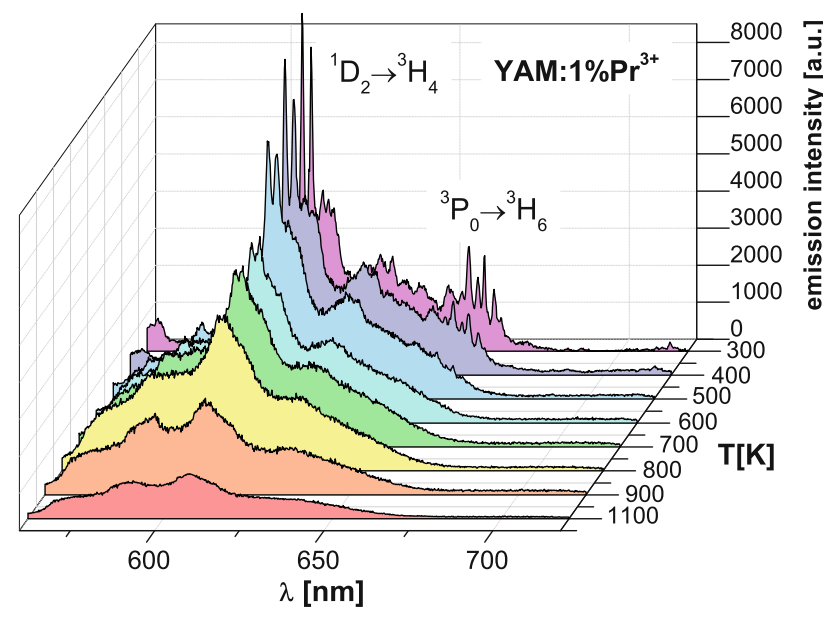

Fig. 3 Emission spectra of $1 \% \mathrm{Pr}^{3+}$ :YAM as a function of temperature after blue ${ }^{3} \mathrm{P}_{2}$ level excitation

${ }^{1} D_{2}$ lines are present in the spectrum and that, as temperature is increased, the overall luminescence intensity decreases and the intensity of ${ }^{1} \mathrm{D}_{2}$ emissions increases relatively to that of the ${ }^{3} \mathrm{P}_{0}$ (at $660 \mathrm{~nm}$ ) one. Figure 3 indicates that temperature quenching of the ${ }^{3} \mathrm{P}_{0}$ emission is stronger than that of the ${ }^{1} \mathrm{D}_{2}$ one.

\subsection{Excited state dynamics}

Decays originating from the ${ }^{3} \mathrm{P}_{0}$ and ${ }^{1} \mathrm{D}_{2}$ levels were measured in the function of activator concentration and temperature. ${ }^{3} \mathrm{P}_{0}$ decays were measured at $541 \mathrm{~nm}$ where an intense emission occurs. This wavelength was chosen as it is spectrally isolated from the ${ }^{1} \mathrm{D}_{2}$ emission. Nonexponentiality of the ${ }^{3} \mathrm{P}_{0}$ and ${ }^{1} \mathrm{D}_{2}$ decays was observed even at low dopant concentrations, and at low temperatures indicating that energy transfer processes strongly contribute to the decays of these two luminescent levels. It was also observed that, as the concentration of $\mathrm{Pr}^{3+}$ ions was increased, the ${ }^{3} \mathrm{P}_{0}$ fluorescence decays shortened and became strongly nonexponential, see Fig. 4. Low temperature ${ }^{3} \mathrm{P}_{0}$ lifetime determined in $0.1 \% \mathrm{Pr}^{3+}$-doped sample from the long time part of the decay was $13 \mu$ s when the ${ }^{1} \mathrm{D}_{2}$ decay in the lowest concentration sample indicated lifetime of $378 \mu$ s which is in agreement with results for other praseodymium-activated oxide crystals like $\mathrm{LaAlO}_{3}$ [14]. When the ${ }^{3} \mathrm{P}_{0}$ decays were registered in long time scale and over several e-foldings, the presence of a weaker, slow component of the decay extending up to about $10 \mathrm{~ms}$ has been observed.

The luminescence decays of the ${ }^{3} \mathrm{P}_{0}$ level in $1 \%$ $\operatorname{Pr}^{3+}: Y A M$ for several different temperatures are plotted in Fig. 5. The decays shorten with increasing temperature, indicating efficient nonradiative quenching. From Fig. 5, it can be also seen that slow component of the ${ }^{3} \mathrm{P}_{0}$ state decays

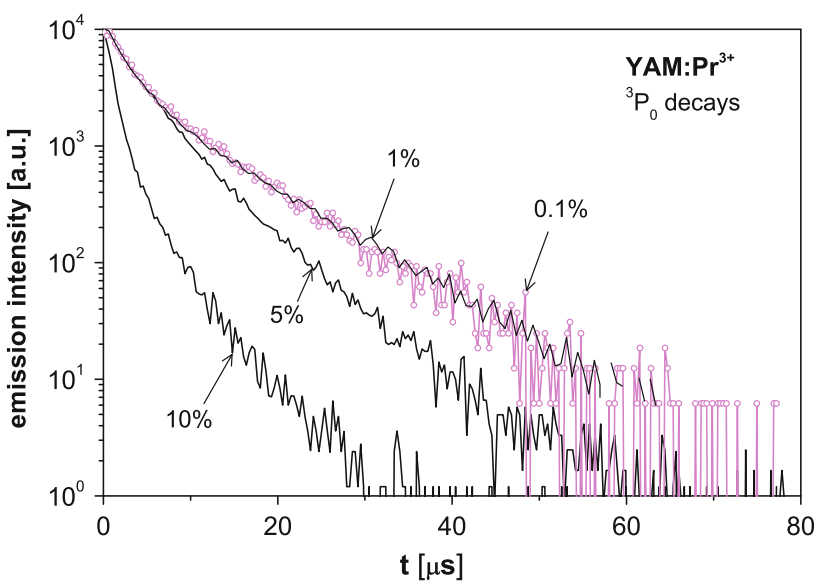

Fig. 4 Plot of the ${ }^{3} \mathrm{P}_{0}$ level decays in $\mathrm{Pr}^{3+}$ :YAM for different activator concentrations, $T=300 \mathrm{~K}$. Excitation of ${ }^{3} \mathrm{P}_{2}$ levels

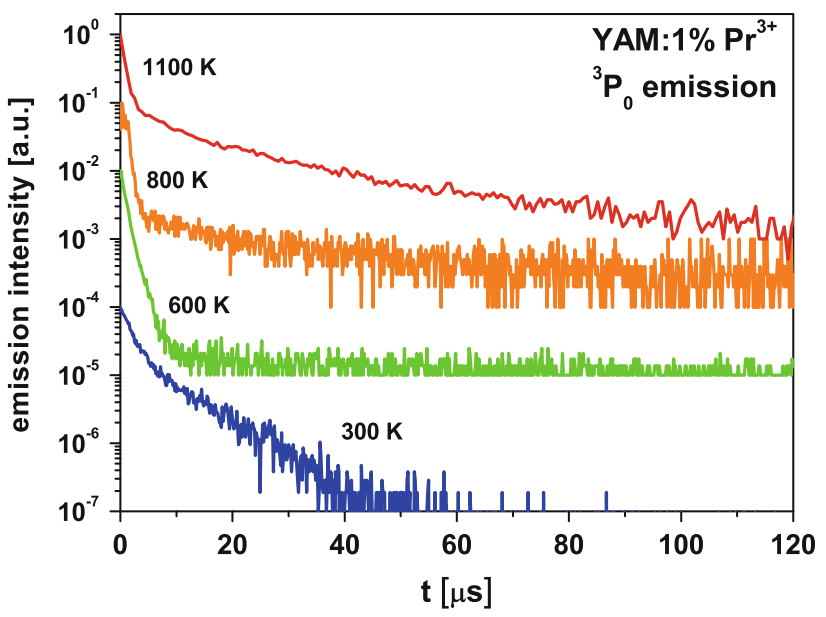

Fig. 5 Plot of the ${ }^{3} \mathrm{P}_{0}$ level decays for different temperatures in $1 \% \mathrm{Pr}^{3+}$ :YAM. Excitation of ${ }^{3} \mathrm{P}_{2}$ levels

becomes stronger with the increase in temperature. The ${ }^{1} \mathrm{D}_{2}$ excited level decays are less temperature influenced up to about $900 \mathrm{~K}$; however, at higher temperatures, these decays become much faster and strongly nonexponential.

\section{Discussion}

In YAM, as well as in some other praseodymium compounds, it is observed that upon photo-excitation of the ${ }^{3} \mathrm{P}_{0}$ level not only emission from the ${ }^{3} \mathrm{P}_{0}$ level is observed, but also from the ${ }^{1} \mathrm{D}_{2}$ one $[15,16]$. Despite the extensive studies on praseodymium systems that have been done and presented in literature conclusions regarding the mechanism of relaxation from ${ }^{3} \mathrm{P}_{0}$ to ${ }^{1} \mathrm{D}_{2}$ level are still not consistent. There are three decay paths of interest possible: radiative, multiphonon relaxation and energy transfer. Radiative transitions from ${ }^{3} \mathrm{P}_{0}$ to ${ }^{1} \mathrm{D}_{2}$ are spin forbidden 
and have very low probability [17]. Also, as these levels are separated by about $3,500 \mathrm{~cm}^{-1}$, the probability of multiphonon process in the system considered here is not significant [18] —of the order of $10^{3} \mathrm{~s}^{-1}$ [17] at room temperature. The $\mathrm{T}$ dependence of multiphonon relaxation rate (MPR) can be written as [19]:

$W_{\mathrm{NR}}(T)=W_{\mathrm{NR}}(0)\left[1-\exp \left(-\frac{\hbar \omega}{k T}\right)\right]^{-p}$

where $p$ is the number of phonons involved in the nonradiative decay $p=\Delta \mathrm{E} / \mathrm{h} \omega$ and $\mathrm{W}_{\mathrm{NR}}(0)$ is the multiphonon decay rate at $0 \mathrm{~K}$, given as

$W_{\mathrm{NR}}(0)=\beta \exp (-\alpha \Delta E)$

where $\Delta \mathrm{E}$ is the energy gap to the next lower energy level, $\alpha$ and $\beta$ are phenomenological parameters. With the use of $\alpha$ and $\beta$ determined for YAG in [17] and taking the maximum phonon energy of the YAM host of $812 \mathrm{~cm}^{-1}$ as determined from Raman scattering data, $\Delta E=3,500 \mathrm{~cm}^{-1}$, the temperature dependence of $\mathrm{W}_{\mathrm{NR}}$ for ${ }^{3} \mathrm{P}_{0} \rightarrow{ }^{1} \mathrm{D}_{2}$ transition was calculated. In the result, at high temperature of $1,300 \mathrm{~K}$, we have $\mathrm{W}_{\mathrm{NR}}(1,300 \mathrm{~K})=3.8 \times 10^{3} \mathrm{~s}^{-1}$ which is still much lower than the ${ }^{3} \mathrm{P}_{0}$-radiative decay rate of $7.7 \times 10^{4} \mathrm{~s}^{-1}$.

Therefore, it is concluded here that for populating the ${ }^{1} \mathrm{D}_{2}$ level and resulting emission energy transfer process is most likely. Also, this energy transfer, called cross-relaxation (CR), is responsible for sharp decrease in the luminescence with increasing activator concentration in most praseodymium compounds. CR process has been first studied by Hegarty et al. [20] and by Vial and Buisson [21] in $\operatorname{Pr}^{3+}: \mathrm{LaF}_{3}$ crystals. It was shown that in praseodymiumactivated solids, concentration quenching of the ${ }^{3} \mathrm{P}_{0}$ fluorescence dynamics is related to the $\mathrm{CR}$ mechanism of the type $\left({ }^{3} \mathrm{P}_{0},{ }^{3} \mathrm{H}_{4}\right) \rightarrow\left({ }^{3} \mathrm{H}_{6},{ }^{1} \mathrm{D}_{2}\right)$. Another $\left({ }^{3} \mathrm{P}_{0},{ }^{3} \mathrm{H}_{4}\right) \rightarrow\left({ }^{1} \mathrm{G}_{4}\right.$, ${ }^{1} \mathrm{G}_{4}$ ) process can be disregarded because of a large energy mismatch of about $800 \mathrm{~cm}^{-1}$ and because both participating transitions are spin forbidden. $\mathrm{Wu}$ et al. [22] reported on the backward process of the type $\left({ }^{1} \mathrm{D}_{2}\right.$, $\left.{ }^{3} \mathrm{H}_{6}\right) \rightarrow\left({ }^{3} \mathrm{H}_{4},{ }^{3} \mathrm{P}_{1}\right)$ which reaches ${ }^{3} \mathrm{P}_{1}$ state and after fast nonradiative relaxation results in refeeding the ${ }^{3} \mathrm{P}_{0}$ state. The energy levels of YAM [6] indicate that this process could excite either the ${ }^{3} \mathrm{P}_{1}$ or directly the ${ }^{3} \mathrm{P}_{0}$ levels depending on the energy of the initial ${ }^{3} \mathrm{H}_{6}$ Stark level.

This agrees well with our observations of the slow component, with the lifetime close to the half of the ${ }^{1} \mathrm{D}_{2}$ one, in the ${ }^{3} \mathrm{P}_{0}$ decay (see Fig. 6) and its increase in intensity with temperature. Discussed mechanisms are presented schematically in Fig. 7, where the $\mathrm{Pr}^{3+}$ pair system has been reduced to the main levels of CR process.

In order to determine the $\mathrm{CR}$ rates and its temperature dependence and to model fluorescence dynamics, the following system of differential rate equations was proposed and numerically solved.

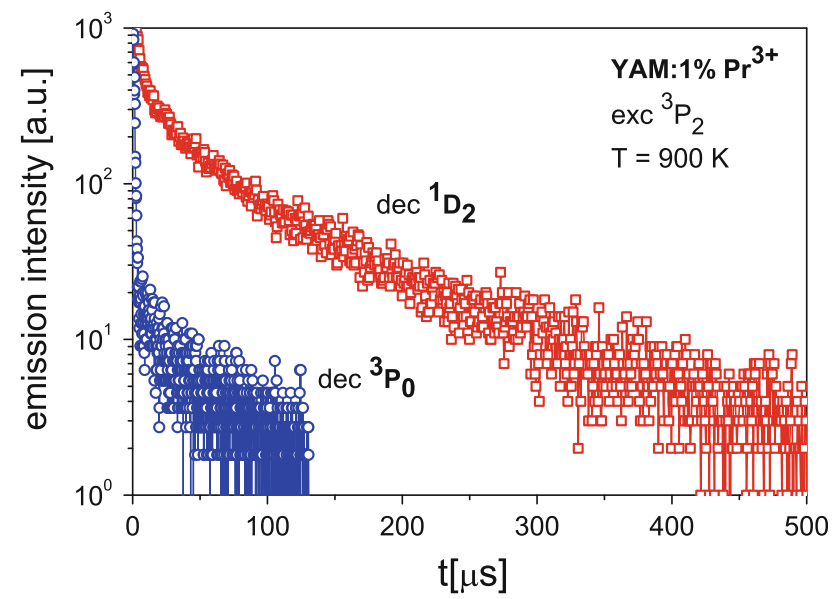

Fig. 6 Plot of the ${ }^{3} \mathrm{P}_{0}$ and ${ }^{1} \mathrm{D}_{2}$ level decays at $900 \mathrm{~K}$ in $1 \% \mathrm{Pr}^{3+}$ :YAM. Excitation of ${ }^{3} \mathrm{P}_{2}$ levels

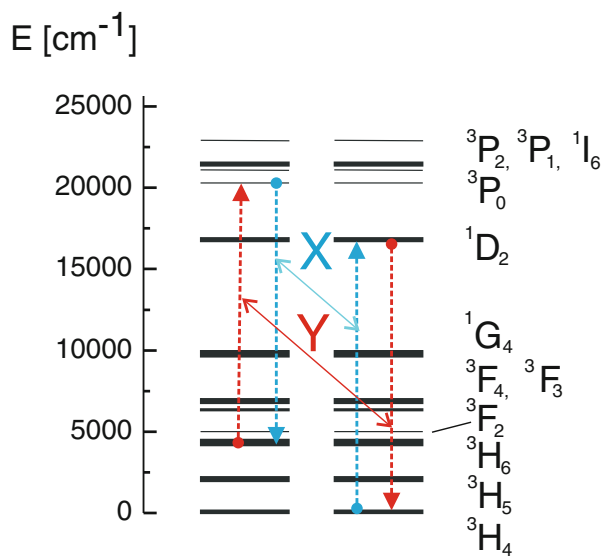

Fig. 7 Scheme of the cross-relaxation process of the type $\left({ }^{3} \mathrm{P}_{0}\right.$, $\left.{ }^{3} \mathrm{H}_{4}\right) \rightarrow\left({ }^{3} \mathrm{H}_{6},{ }^{1} \mathrm{D}_{2}\right)$ responsible for populating the ${ }^{1} \mathrm{D}_{2}$ energy level of $\mathrm{Pr}^{3+}$ ion after ${ }^{3} \mathrm{P}_{0}$ excitation, the backward process $\left({ }^{1} \mathrm{D}_{2}\right.$, $\left.{ }^{3} \mathrm{H}_{6}\right) \rightarrow\left({ }^{3} \mathrm{H}_{4},{ }^{3} \mathrm{P}_{0}\right)$ is also shown

$$
\begin{aligned}
\frac{\mathrm{d} n_{3}}{\mathrm{~d} t}= & -x n_{3} n_{0}+y n_{2} n_{1}-\left(W_{32}+A_{30}+A_{31}+A_{32}\right) n_{3} \\
\frac{\mathrm{d} n_{2}}{\mathrm{~d} t}= & \left(W_{32}+A_{32}\right) n_{3}-\left(W_{21}+A_{21}+A_{20}\right) n_{2} \\
& +x n_{3} n_{0}-y n_{2} n_{1} \\
\frac{\mathrm{d} n_{1}}{\mathrm{~d} t}= & \left(W_{21}+A_{20}+A_{21}\right) n_{2}-\left(W_{10}+A_{10}\right) n_{1} \\
& +x n_{3} n_{0}-y n_{2} n_{1}+A_{31} n_{3} \\
\frac{\mathrm{d} n_{0}}{\mathrm{~d} t}= & -x n_{3} n_{0}+y n_{2} n_{1}+\left(W_{10}+A_{10}\right) n_{1} \\
& +A_{20} n_{2}+A_{30} n_{3}
\end{aligned}
$$

where $n_{0}, n_{1}, n_{2}$ and $n_{3}$ are the populations of the ${ }^{3} \mathrm{H}_{4},{ }^{3} \mathrm{H}_{6}$, ${ }^{1} \mathrm{D}_{2}$ and ${ }^{3} \mathrm{P}_{0}$ levels of $\mathrm{Pr}^{3+}$, respectively (units of $\mathrm{cm}^{-3}$ ), $\mathrm{x}$ and $\mathrm{y}$ are forward and backward $\mathrm{CR}$ transfer rates, respectively (units of $\mathrm{s}^{-1} \mathrm{~cm}^{3}$ ), $A_{i k}$ are the radiative transition probabilities between the $i$ and $k$ states, $W_{i}$ is the phonon de-excitation rate for the $i$-th level (units of $\mathrm{s}^{-1}$ ). 
As only the mentioned four levels are involved, we have $n_{1}+n_{2}+n_{3}+n_{4}=N$, where $N$ is $\operatorname{Pr}^{3+}$ concentration in the sample (units of $\mathrm{cm}^{-3}$ ).

Also, $A_{i k}=\beta_{i k} A_{i}$, where $\beta_{i k}$ are the branching ratios and $A_{\mathrm{i}}$ radiative transition probabilities of the ${ }^{3} \mathrm{P}_{0}(i=3)$ and ${ }^{1} \mathrm{D}_{2}(i=2)$ states. Thus, for evaluating $A_{3 k}$ and $A_{2 k}$ (where $k=0.1)$ values, the corresponding branching ratios were obtained from the normalized fluorescence spectra by comparing the relative areas under the emission peaks. It was also assumed that $A_{32}=0$ and $W_{21}=0$ which means that ${ }^{3} \mathrm{H}_{6}$ is only populated by radiative transitions from ${ }^{3} \mathrm{P}_{0}$ and ${ }^{1} \mathrm{D}_{2}$ levels. No value of the ${ }^{3} \mathrm{H}_{6}$ state lifetime is reported for praseodymium-activated oxide hosts. Wu et al. [22] assumed in their calculations for Pr:YAG the ${ }^{3} \mathrm{H}_{6}$ lifetime of $5 \mathrm{~ms}$, that is, the decay rate of $2 \times 10^{2} \mathrm{~s}^{-1}$. However, because of the small energy gap of the order of $1,200 \mathrm{~cm}^{-1}$ [6] to the ${ }^{3} \mathrm{H}_{5}$ level, the highest limit of the decay rate of ${ }^{3} \mathrm{H}_{6}$ level $\left(W_{1}+A_{1}\right)$ is estimated to be about $10^{6} \mathrm{~s}^{-1}$. Because ${ }^{1} \mathrm{D}_{2}$ decay is temperature dependent, effective lifetime values of the ${ }^{1} D_{2}$ level experimentally determined for each temperature were used in our calculations. Forward and backward transfer rates ( $\mathrm{x}$ and $\mathrm{y}$, respectively) were treated as adjustable parameters. Values of most parameters necessary to solve the above indicated system of equations have been experimentally determined and are listed in Table 1.

From the presented model of CR transitions, it may be concluded that the dynamics of ${ }^{3} \mathrm{P}_{0}$ excited state is strongly affected by both forward and backward transfer rates as well as by the lifetime of ${ }^{1} \mathrm{D}_{2}$ level. Because of the relatively fast relaxation, the influence of ${ }^{3} \mathrm{H}_{6}$ level on ${ }^{3} \mathrm{P}_{0}$ decay curve can be excluded. Simple numerical solutions showing influence of $x$ and $y$ transfer rates on dynamics of ${ }^{3} \mathrm{P}_{0}$ level are presented in Fig. 8. As could be seen, the $\mathrm{x}$ factor is responsible for quenching of the luminescence from ${ }^{3} \mathrm{P}_{0}$ state, resulting in shortening of its lifetime when

Table 1 Summary of the parameter values used to solve numerically the population equation system (3), see text for identification of constants

\begin{tabular}{lll}
\hline Level & Parameter & Value \\
\hline${ }^{3} \mathrm{P}_{0}$ & $\mathrm{~A}_{3}$ & $7.5 \times 10^{4} \mathrm{~s}^{-1}$ \\
& $\beta_{30}\left({ }^{3} \mathrm{P}_{0} \rightarrow{ }^{3} \mathrm{H}_{4}+{ }^{3} \mathrm{H}_{5}\right)$ & 0.7 \\
& $\beta_{31}\left({ }^{3} \mathrm{P}_{0} \rightarrow{ }^{3} \mathrm{H}_{6} \cdots{ }^{1} \mathrm{G}_{4}\right)$ & 0.3 \\
& $\beta_{32}\left({ }^{3} \mathrm{P}_{0} \rightarrow{ }^{1} \mathrm{D}_{2}\right)$ & 0 \\
& $\mathrm{~W}_{32}$ & $2 \times 10^{3} \mathrm{~s}^{-1}$ \\
${ }^{1} \mathrm{D}_{2}$ & $\mathrm{~A}_{2}$ & $2.6 \times 10^{3} \mathrm{~s}^{-1}$ \\
& $\beta_{20}\left({ }^{1} \mathrm{D}_{2} \rightarrow{ }^{3} \mathrm{H}_{4}+{ }^{3} \mathrm{H}_{5}\right)$ & 0.6 \\
& $\beta_{21}\left({ }^{1} \mathrm{D}_{2} \rightarrow{ }^{3} \mathrm{H}_{6} \cdots{ }^{1} \mathrm{G}_{4}\right)$ & 0.4 \\
& $\mathrm{~W}_{21}$ & 0 \\
${ }^{3} \mathrm{H}_{6}$ & $\mathrm{~W}_{10}$ & $2 \times 10^{5} \mathrm{~s}^{-1}$ \\
& $\mathrm{~A}_{10}$ & 0 \\
\hline
\end{tabular}
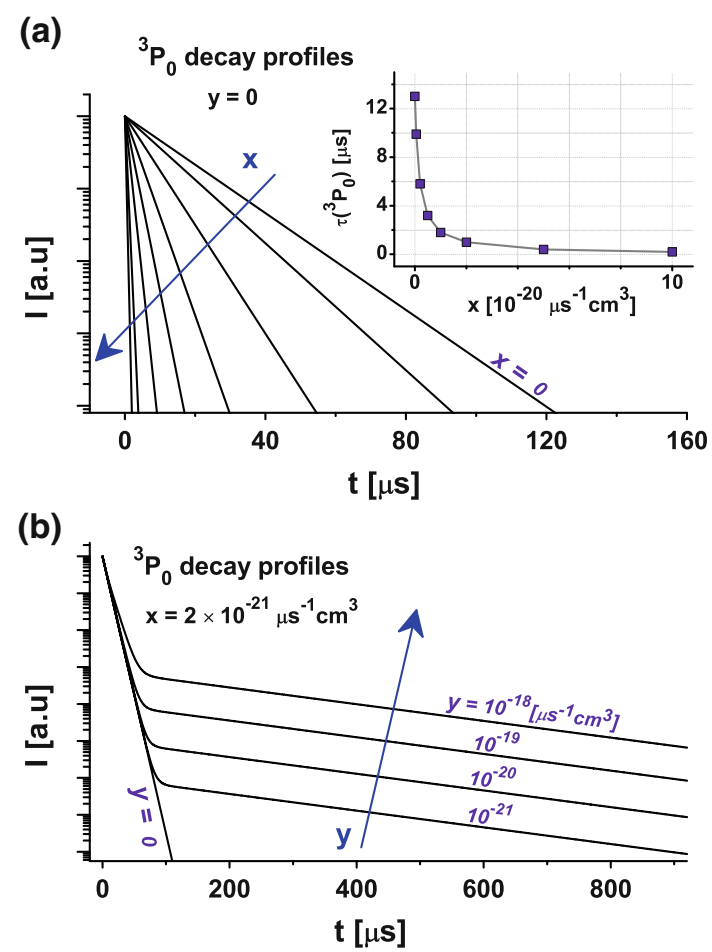

Fig. 8 Decay profiles of ${ }^{3} \mathrm{P}_{0}$ level calculated from the differential equations system (3): a For different forward CR transfer rates$x$ (for $y=0 \mu \mathrm{s}^{-1} \mathrm{~cm}^{3}$ ). Inset presents dependence of the estimated ${ }^{3} \mathrm{P}_{0}$ lifetime on $x$. Each data point in the inset corresponds to one decay profile. b For different backward CR transfer rates-y (for $x=2 \times 10^{-21} \mu^{-1} \mathrm{~cm}^{3}$ ). The short and the long time part of the decay curves represents the lifetime of ${ }^{3} \mathrm{P}_{0}$ state and the half of ${ }^{1} \mathrm{D}_{2}$ level lifetime, respectively

the y process activates the long part on temporal characteristic with decay constant equal to half of ${ }^{1} \mathrm{D}_{2}$ lifetime.

The next step in our investigation was to determine the ${ }^{3} \mathrm{P}_{0}$ CR rates for $1 \%$ doped sample, $X$ and $Y$ with units of $\mathrm{s}^{-1}$, and their temperature dependence. $X$ and $Y$ are related to $x$ and $y$ as follows: $X, Y=x, y \times N$, where $N$ equals $2 \times 10^{20} \mathrm{~cm}^{-3}$ for 1 at. $\%$ doped YAM sample [23].

Our analysis indicated that the short-time exponential part of the decays represents the ${ }^{3} \mathrm{P}_{0}$ lifetime; thus, crossrelaxation transfer rates could be simply calculated as $X=1 / \tau-1 / \tau_{0}$, where $\tau$ is the fluorescence decay time, and $\tau_{0}$ is the isolated ion lifetime of $13 \mu$ s measured in the lowest concentration sample. This value is close to the value of $16 \mu$ s determined in [6] for the praseodymium B site in YAM. It should also be noted that in our experiments at room and higher temperatures, no emission lineshapes nor fluorescence dynamics dependence on excitation wavelengths, which may result from the multisite character of YAM, were observed.

The determined quenching rates $X$ are plotted as a function of temperature for $1 \% \mathrm{Pr}^{3+}$ :YAM in Fig. 9. It is observed that the cross-relaxation rate remains practically 
constant up to about $180 \mathrm{~K}$ and starts to increase rapidly at higher temperatures. As the ${ }^{3} \mathrm{P}_{0}$ radiative decay rate is independent on temperature and phonon-assisted relaxation is weak, with the cutoff phonon frequency of YAM equal $812 \mathrm{~cm}^{-1}$ about 4 phonons are required to bridge the ${ }^{3} \mathrm{P}_{0} \rightarrow{ }^{1} \mathrm{D}_{2}$ energy gap, then this dependence results from thermal activation of Stark levels participating in the forward CR process, thus influencing the spectral overlap between the donor and acceptor transitions. However, temperature dependencies of the shape, peak intensities and widths of participating transition could also be considered. Such thermally activated process normally obeys an Arrhenius-type temperature dependence $X(0) \exp (-\Delta E / \mathrm{kT})$, where $\Delta E$ is an activation energy [24]. Thus, we attempted to fit the experimental results presented in Fig. 9 with this relation. The best fit was obtained with $\Delta E=680 \mathrm{~cm}^{-1}$ at high temperatures and $\Delta E=3 \mathrm{~cm}^{-1}$ at cryogenic temperatures. The energy level positions presented in [6] demonstrate that for both forward and backward process, resonant or quasi-resonant $\mathrm{CR}$ transitions initiate from the higher-lying Stark sublevels in the ${ }^{3} \mathrm{H}_{4}$ and ${ }^{3} \mathrm{H}_{6}$ multiplet, respectively. The activation energy $\Delta E=680 \mathrm{~cm}^{-1}$ obtained from the fitting is close to the value of $684 \mathrm{~cm}^{-1}$ of the Stark level in the ${ }^{3} \mathrm{H}_{4}$ multiplet [6]. Thus, the responsible CR process could be $\left({ }^{3} \mathrm{P}_{0}\left(20,725 \mathrm{~cm}^{-1}\right)\right.$, ${ }^{3} \mathrm{H}_{4}\left(684 \mathrm{~cm}^{-1}\right) \rightarrow\left({ }^{3} \mathrm{H}_{6}\left(4,942 \mathrm{~cm}^{-1},{ }^{1} \mathrm{D}_{2} \quad\left(16,464 \mathrm{~cm}^{-1}\right)\right.\right.$ with a small mismatch of $3 \mathrm{~cm}^{-1}$. It shows that the temperature dependence of forward process is well explained in terms of resonance conditions. The room temperature $X$ rate value in $1 \% \operatorname{Pr}^{3+}$ sample is $3 \times 10^{-2} \mu \mathrm{s}^{-1}$ and is close to the values determined for YAG [22] and $\mathrm{LaAlO}_{3}$ [14] crystals.

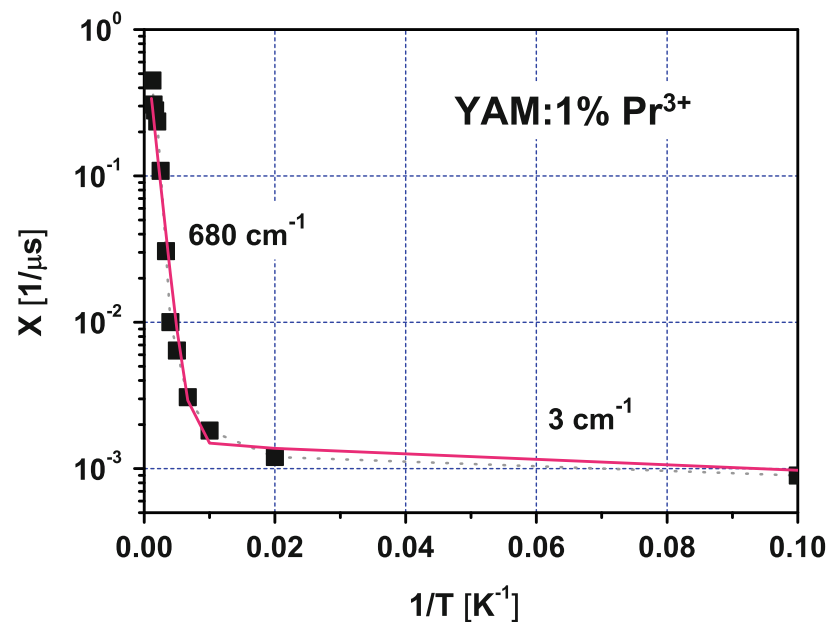

Fig. 9 Temperature dependence of the $X$ forward cross-relaxation rates for $\left({ }^{3} \mathrm{P}_{0},{ }^{3} \mathrm{H}_{4}\right) \rightarrow\left({ }^{3} \mathrm{H}_{6},{ }^{1} \mathrm{D}_{2}\right)$ in $1 \% \mathrm{Pr}^{3+}$ :YAM. The squares are the experimental data, and the solid line indicates calculated thermal dependence

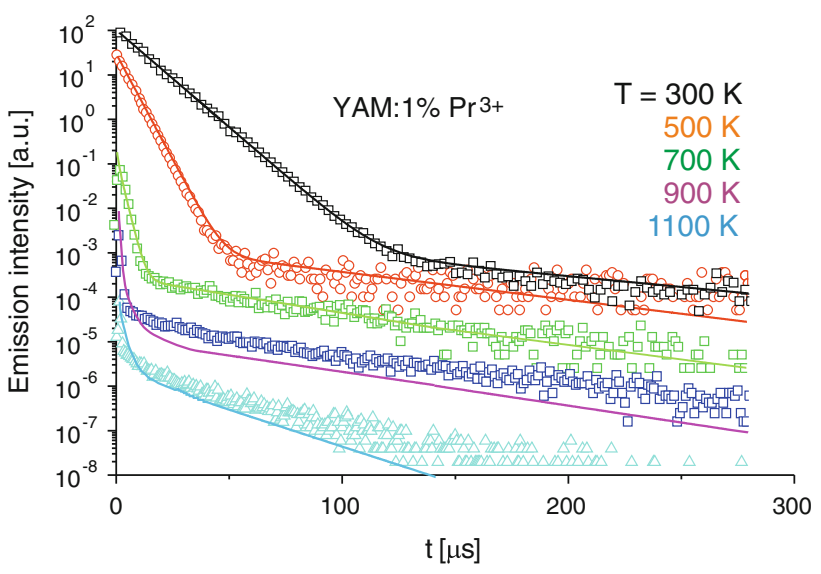

Fig. 10 Decay profiles of ${ }^{3} \mathrm{P}_{0}$ exited state in $1 \% \mathrm{Pr}^{3+}$ :YAM for different temperatures. The points are the experimental data, the solid lines are the fits resulting from the solutions of rate equations (3) taking into consideration the forward $(X)$ and backward $(Y)$ energy transfers

The backward CR transfer is represented by the long time part of ${ }^{3} \mathrm{P}_{0}$ decays. By fitting the solutions of rate equations to the experimentally determined decay curves, the values of $\mathrm{Y}$ for different temperatures were obtained. For example, the room temperature $\mathrm{Y}$ rate value in $1 \%$ $\mathrm{Pr}^{3+}$ sample is $3.2 \times 10^{-1} \mu \mathrm{s}^{-1}$ when it is $1.8 \mu \mathrm{s}^{-1}$ and $35 \mu \mathrm{s}^{-1}$ at $500 \mathrm{~K}$ and $700 \mathrm{~K}$, respectively. In Fig. 10, comparison of the calculated data with experimental points is presented. We obtained generally good fit for temperatures up to about $700 \mathrm{~K}$, however, at higher temperatures our model did not reproduce the experimental results well. This may be due to the fact that at higher temperatures, the multiphonon relaxation rate from ${ }^{3} \mathrm{P}_{0}$ increases and that the energy migration between $\mathrm{Pr}^{3+}$ ions is more likely to occur. It could be also interpreted in terms of energy dissipation between several $\mathrm{Pr}^{3+}$ sites in YAM at higher temperatures which increases the number of resonant and quasi-resonant $\mathrm{CR}$ transitions.

It is observed that for temperatures above about $200 \mathrm{~K}$ the backward transfer rate values are about one order of magnitude higher than those for the forward process. The temperature dependence of backward process is explained in terms of activation of higher crystal-field levels in the ${ }^{3} \mathrm{H}_{6}$ multiplet.

Finally, it must be also noted that the observed shortening of the ${ }^{1} \mathrm{D}_{2}$ decays with increasing temperature could be related to the increase in the back transfer, rather than ${ }^{1} \mathrm{D}_{2}$ cross-relaxation itself, similar situation has been reported in [25] for Pr-doped $\mathrm{CsCdBr}_{3}$.

\section{Summary}

Fluorescence spectra and fluorescence lifetimes corresponding to transitions from the ${ }^{3} \mathrm{P}_{0}$ level of praseodymium 
in YAM have been measured and analyzed. We have identified and characterized a nonradiative cross-relaxation channels from the ${ }^{3} \mathrm{P}_{0}$ manifold concluding that a considerable portion of the overall fluorescence emission stems from the ${ }^{1} \mathrm{D}_{2}$ manifold. Also, the shortening and nonexponentiality of the decays, observed with increasing activator concentrations and temperature, were interpreted in terms of cross-relaxation among the $\mathrm{Pr}^{3+}$ ions. Crossrelaxation rates were experimentally determined as a function of temperature in a wide temperature range from 10 to $1,000 \mathrm{~K}$ and used for modeling of the decays with standard rate equation technique. Influence of the forward and the backward CR processes, and their temperature dependence, on ${ }^{3} \mathrm{P}_{0}$ decay shape was discussed. The present results give insight into factors involved in the CR quenching in materials activated with $\mathrm{Pr}^{3+}$ ions. Described approach is planned to be used to investigate $\operatorname{Pr}^{3+}{ }^{3} \mathrm{P}_{0}$ cross-relaxation in other hosts.

This information could be helpful in the development of praseodymium-based phosphors for fluorescence thermometry utilizing the intensity ratio method or the lifetime decay method.

Acknowledgments This work was supported by the MNiSzW N N515 081537 project.

Open Access This article is distributed under the terms of the Creative Commons Attribution License which permits any use, distribution, and reproduction in any medium, provided the original author(s) and the source are credited.

\section{References}

1. A.A. Kaminskii, Advanced Solid-State Lasers (New Orleans, LA, 1993) vol 15, ed by A. Pinto, T.Y. Fan (Optical Society of America, Washington DC, 1993), p. 266
2. P. Boutinaud, E. Pinel, M. Oubaha, R. Mahiou, E. Cavalli, M. Bettinelli, Opt. Mater. 28, 9 (2006)

3. M. Nikl, P. Bohacek, A. Vedda, M. Fasoli, J. Pejchal, A. Beitlerova, M. Fraternali, M. Livan, J. Appl. Phys. 104, 093514 (2008)

4. B.M. van der Ende, L. Aartsa, A. Meijerink, Phys. Chem. Chem. Phys. 11, 11081 (2009)

5. T. Gun, P. Metz, G. Huber, Appl. Phys. Lett. 99, 181103 (2011)

6. Y. Rabinovitch, O.K. Moune, D. Tetard, M.D. Faucher, J. Phys. Chem. A 108, 8244 (2004)

7. Y. Huang, K. Jang, X. Wang, G. Xian, E. Cho, H.S. Lee, S.H. Ki, J. Non-Cryst, Solids 353, 4102 (2007)

8. I.R. Mitchell, P.M. Farrell, G.W. Baxter, S.F. Collins, K.T.V. Grattan, T. Su, Rev. Sci. Instrum. 71, 100 (2000)

9. I. Kamma, P. Kommidi, B.R. Reddy, Phys. Status Solidi C 6, S187 (2009)

10. J. Jordan, D.A. Rothamer, Thermographic phosphor based planar thermometry using the trivalent praseodymium ion $\left(\mathrm{Pr}^{3+}\right)$ doped into a yttrium aluminum garnet (YAG) crystal, Spring Technical Meeting of the Central States Section of the Combustion Institute (April 22-24, 2012)

11. D.H. Yoon, I. Yonenaga, N. Ohnishi, T. Fukuda, J. Crystal Growth 142, 339 (1994)

12. D.A. Pawlak, Y. Kagamitani, A. Yoshikawa, K. Wozniak, H. Sato, H. Machida, T. Fukuda, J. Crystal Growth 226, 341 (2001)

13. C.D. Brandle, H. Steinfink, Inorg. Chem. 8, 1320 (1966)

14. M. Malinowski, M. Kaczkan, S. Turczynski, D. Pawlak, Opt. Mater. 33, 1004 (2011)

15. S. Mahlik, M. Malinowski, M. Grinberg, Opt. Mater. 33, 1525 (2011)

16. H. Chen, R. Lian, M. Yin, L. Lou, W. Zhang, S. Xia, J.C. Krupa, J. Phys. Condens. Matter 13, 1151 (2001)

17. A.A. Kaminskii, Laser crystals, their physics and properties (Springer, Berlin, 1981)

18. T.T. Basiev, YuV Orlovskii, K.K. Pukhov, F. Auzel, Laser Phys. 7, 1139 (1997)

19. L.A. Riseberg, H.W. Moos, Phys. Rev. 174, 429 (1968)

20. J. Hegarty, D.L. Huber, W.M. Yen, Phys. Rev. B 25, 5638 (1982)

21. J.C. Vial, R. Buisson, J. Phys. Lett.-Paris 43, L-745-753 (1982)

22. X. Wu, W.M. Dennis, W.M. Yen, Phys. Rev. B 50, 6589 (1994)

23. H. Yamane, M. Omori, T. Hirai, J. Mater. Sci. Lett. 14, 470 (1995)

24. S. Bhushan, M.V. Chukichev, J. Mater. Sci. Lett. 9, 319 (1988)

25. Y. An, P.S. May, J. Lumin. 118, 147 (2006) 